\title{
Empatia Afetiva e Cognitiva no Transtorno do Espectro Autista (TEA): UMA REVISÃo INTEGRATIVA DA LITERATURA ${ }^{1}$

\author{
Affective and Cognitive EMPathy on the Autism SPeCtrum Disorder \\ (ASD): AN INTEGRATIVE LITERATURE REVIEW
}

\author{
Sarah Aline ROZA² \\ Sandra Regina Kirchner GUIMARÃES 3
}

\begin{abstract}
RESUMO: A literatura vem apontando um crescente interesse pelos estudos sobre como a empatia, em seus componentes cognitivo e afetivo, desenvolve-se em pessoas com Transtorno do Espectro Autista (TEA). Assim, esta revisáo integrativa da literatura teve o objetivo de investigar a empatia, em seus componentes cognitivo e afetivo, em indivíduos com TEA e fatores associados. Para isso, efetuou-se uma busca por artigos científicos nas bases de dados PubMed, ERIC e PsycINFO. Adotaram-se as palavras-chave e marcadores booleanos Autism [or] ASD (Autism Spectrum Disorder) [and] Empathy. Como resultado, foram recuperadas 180 publicações, das quais 26 foram analisadas. Os critérios de inclusão foram estudos empíricos - correlacionais e de intervenção que abordassem as relaçóes entre empatia e TEA, indexados nas bases de dados aqui mencionadas entre os anos de 2005 e 2020. As categorias resultantes da análise foram: empatia afetiva e cognitiva, diferenças associadas ao gênero na empatia e diferenças associadas à idade e ao Quociente de inteligência (QI) em pessoas com TEA. Verificou-se que a empatia cognitiva, que envolve a inferência de emoção do estado mental de outra pessoa, pode ser reduzida em pessoas com TEA, enquanto a empatia afetiva, que se refere à capacidade de compartilhar a emoção dos outros, não apresenta déficit. Além disso, alguns estudos apontaram para a importância do ensino de responsividade empática em crianças com TEA. Contudo, não foram encontrados artigos em bases brasileiras sobre o tema, assinalando uma importante lacuna de pesquisas sobre a empatia em pessoas com autismo no cenário nacional.
\end{abstract}

PALAVRAS-CHAVE: Autismo. Empatia. Empatia afetiva. Empatia cognitiva.

\begin{abstract}
Literature has shown a growing interest in studies on how empathy, regarding its cognitive and affective components, can be developed in people with Autism Spectrum Disorder (ASD). Thus, this integrative literature review aimed to investigate empathy, in its cognitive and affective components, in individuals with TEA and associated factors. For this, a search for scientific papers was carried out in the PubMed, ERIC, and PsycINFO electronic databases. The keywords and Boolean operators Autism [or] ASD (Autism Spectrum Disorder) [and] Empathy were adopted. As a result, 180 publications were found, and 26 were analyzed. The inclusion criteria were empirical pieces of research - correlational and intervention - that addressed the relationship between empathy and ASD, indexed in the databases already mentioned between 2005 and 2020. The categories resulting from the investigation were: affective and cognitive empathy, gender differences in empathy, and differences related to age and Intelligence Quotient (IQ) in individuals with ASD. The studies showed that cognitive empathy, which involves the inference of emotion from another person's mental state, can be reduced in people with ASD, while affective empathy, which refers to the ability to share the emotions of others, does not suffer the same deficit. In addition, some studies have pointed to the importance of teaching empathic responsiveness in children with ASD. No studies were found in Brazilian databases on the topic, signaling an important research gap on empathy in people with autism on the national scene.
\end{abstract}

KEYWORDS: Autism. ASD. Empathy. Affective empathy. Cognitive empathy.

\footnotetext{
${ }^{1}$ https://doi.org/10.1590/1980-54702021v27e0028

${ }^{2}$ Doutoranda e Mestra em Educação na linha Processos Psicológicos em contextos Educacionais pela Universidade Federal do Paraná (UFPR). Psicóloga pela Universidade Tuiuti do Paraná (UTP). Licenciada em Letras Português-Inglês pela Universidade Tecnológica Federal do Paraná (UTFPR). Professora de Língua Portuguesa no Colégio da Polícia Militar do Paraná (COM). Curitiba/Paraná/Brasil. E-mail: sarah.a.roza@gmail.com. ORCID: https://orcid.org/0000-0002-7777-7155

${ }^{3}$ Professora permanente do Programa de Pós-Graduação em Educação da Universidade Federal do Paraná (UFPR). Psicóloga pela Pontifícia Universidade Católica do Paraná (PUCPR). Mestra em Educação pela Universidade Federal do Paraná (UFPR). Doutora em Psicologia Escolar e do Desenvolvimento Humano pela Universidade de São Paulo (USP). Curitiba/Paraná/Brasil. E-mail: srkguimaraes@uol.com.br. ORCID: http://orcid.org/0000-0003-3441-0983
} 


\section{INTRODUÇÁo}

O Transtorno do Espectro Autista (TEA) envolve dificuldades na interação social e na comunicação bem como a presença de comportamentos restritos e repetitivos (Associação Americana de Psiquiatria [APA], 2014). Essas adversidades podem representar desafios específicos para famílias, educadores e profissionais que atuam com TEA, uma vez que as dificuldades na interação social têm sido uma das características definidoras dos indivíduos com autismo (Fletcher-Watson et al., 2014). Nesse contexto, estudos vêm apontando que pessoas diagnosticadas com TEA demonstram falta de empatia como uma das características centrais de comportamento (Decety \& Jackson, 2004; Dziobek et al., 2008; Gaigg, 2012).

A empatia é descrita como uma habilidade que permite a uma pessoa entender e compartilhar o estado emocional de outro indivíduo, incluindo dois componentes: um cognitivo e outro afetivo (Davis, 1980). A empatia cognitiva é a capacidade de compreender e prever o comportamento de outras pessoas em termos de estados mentais atribuídos, tais como: acreditar, saber, entender e adivinhar (Blair, 2005). A empatia afetiva corresponde a uma resposta emocional que se origina em um indivíduo e é paralela ao estado emocional de outro indivíduo; trata-se de uma resposta afetiva que é mais apropriada à situação da outra pessoa (Hoffman, 2000). A empatia (cognitiva e afetiva) guarda relação com a Teoria da Mente (também referida como ToM - sigla de Theory of Mind), que diz respeito à capacidade de atribuir e representar em si próprio e em outras pessoas os estados mentais independentes, como seus pensamentos, seus desejos, suas crenças e suas emoçóes (Domingues \& Maluf, 2008). Por isso, é possível dizer que a Teoria da Mente influencia o desenvolvimento de outras habilidades sociocognitivas, entre elas, a empatia, embora, nem sempre, a associação entre a aquisição de uma Teoria da Mente e a habilidade de compartilhar emoçóes seja comprovada - como é o caso do estudo de Pavarini e Souza (2010), cujos resultados não mostraram correlação entre o desempenho de crianças pré-escolares nas tarefas de Teoria da Mente e o grau de empatia.

A empatia requer, essencialmente, componentes cognitivo e afetivo, pois é necessário que se identifique os pensamentos e as emoçóes de outra pessoa, respondendo a esses estados com uma emoção apropriada (Decety \& Jackson, 2004). De acordo com Vignemont e Singer (2006), há empatia se: a) a pessoa está em um estado afetivo; b) esse estado é isomórfico ao estado afetivo de outra pessoa; c) esse estado é provocado pela observação ou imaginação do estado afetivo de outra pessoa; d) sabe-se que a outra pessoa é a fonte do seu próprio estado afetivo. Entretanto, é importante salientar que a empatia se refere a adotar automaticamente o estado emocional ou cognitivo de alguém, mantendo a distinção eu-outro (Decety \& Jackson 2004).

O componente cognitivo da empatia exige a ativação de funçóes cognitivas complexas, como a tomada de perspectiva e a mentalização (Shamay-Tsoory, 2011), enquanto a empatia afetiva inclui o compartilhamento de experiências relativas aos estados internos de outra pessoa (Zaki \& Ochsner, 2012). Nessa perspectiva, alguns estudos mencionam a possibilidade de trabalhar com pessoas com TEA visando o desenvolvimento da empatia (Blair, 2005; Holopainen et al., 2018; Shamay-Tsoory, 2011).

Indivíduos diagnosticados com TEA apresentam, frequentemente, problemas e limitaçôes na compreensão de estados mentais de outras pessoas, como pensamentos, intençóes e crenças (Gaigg, 2012). Nesse sentido, pesquisas têm apontado que pessoas com TEA não exi- 
bem apenas dificuldades em atribuir e compreender os estados mentais e emocionais de outras pessoas, mas também de responder aos estados mentais de outras pessoas com respostas emocionais apropriadas (Sucksmith et al., 2013). Prejuízos na empatia foram relatados em crianças e adultos com TEA (Sucksmith et al., 2013), bem como em indivíduos que, embora diagnosticados dentro do espectro autista, apresentam altos níveis de inteligência (Baron-Cohen \& Wheelwright, 2004; Lombardo et al., 2007).

Pesquisas que versam sobre a empatia têm levantado uma hipótese de desiquilíbrio dessa habilidade em pessoas com TEA (Smith, 2009). Nessa perspectiva, estudos sugerem que, em vez de um déficit global de empatia, apenas o componente cognitivo estaria comprometido em indivíduos com autismo, podendo, ainda, o componente afetivo ser elevado, em decorrência de uma hiperexcitação em resposta a pistas emocionais de outras pessoas, como expressóes faciais de medo (Moriwaki et al., 2011). Embora o déficit em empatia cognitiva venha sendo apoiado de forma recorrente por pesquisas envolvendo pessoas com TEA, com ou sem Quociente de Inteligência (QI) elevado (Dziobek et al., 2008; Rogers et al., 2007), a empatia afetiva ainda carece de mais investigação.

O prejuízo na interação social figura como uma das características associadas ao déficit de empatia em indivíduos com TEA (Mul et al., 2018). Contudo, essa associação ainda apresenta inconsistência, pois o comprometimento da empatia em indivíduos com autismo pode ser regulado por diferentes fatores, já que a empatia é multidimensional (Decety \& Jackson, 2004) e tem relação com o contexto (Powell \& Roberts, 2017). Dessa forma, torna-se imprescindível lançar luz e revisar sistematicamente o status da pesquisa envolvendo a empatia em indivíduos com TEA, tendo em vista que é um campo fértil, com pesquisas atuais e de relevância para educadores, psicólogos e pesquisadores de diversas áreas.

É nesse contexto que este estudo de revisão se insere, tendo como objetivos: (a) verificar como os componentes cognitivo e afetivo são abordados nas pesquisas selecionadas; (b) investigar como os constructos de empatia foram medidos nas pesquisas selecionadas; e (c) elencar outras categorias de análise dos estudos que possam elucidar as características da empatia em pessoas com TEA.

\section{Método}

Nesta seção, serão abordados o tipo de estudo realizado, as bases indexadas, os descritores, os critérios de inclusão e de exclusão e os procedimentos utilizados e, por fim, como se deu a análise de dados.

\subsection{Tipo De estudo}

O estudo aqui apresentado consiste em uma revisão integrativa da literatura científica. Para isso, realizou-se uma busca abrangente de pesquisas na interface entre as áreas da Saúde, Psicologia e Educação Especial. A operacionalização da revisão fundamentou-se nos procedimentos empregados por Mendes et al. (2008), que seguem uma metodologia que focaliza alguns pontos: a delimitação do tema, a proposição de critérios de exclusão/inclusão, a 
organização de categorias de análise, a verificação das pesquisas elencadas, a análise sintética dos principais achados e o mapeamento do tema de forma atual.

A categorização e a verificação dos achados foram elaboradas por duas pesquisadoras de forma independente. Nas bases PsycINFO, ambas encontraram 124 artigos; na PubMed, as pesquisadoras obtiveram os mesmos resultados, com um total de 25 artigos; entretanto, na base $E R I C$, houve diferença no resultado: uma das pesquisadoras encontrou 35 artigos, enquanto a outra encontrou 31. Apesar disso, os quatro artigos encontrados a mais não foram incluídos na revisão, por não se enquadrarem nos critérios de inclusão desta pesquisa. Posto isso, o tema que guiou esta revisão foi a empatia, em seus componentes cognitivo e afetivo, em indivíduos com TEA e fatores associados.

\subsection{BASES INDEXADAS E DESCRITORES UTILIZADOS}

Para a realização desta revisão, organizou-se uma busca sistemática e abrangente nas seguintes bases de dados eletrônicas: PubMed (Biblioteca Nacional de Medicina dos Estados Unidos), ERIC (Centro de Informação de Recursos Educacionais) e na PsycINFO (Base de dados no campo da Psicologia, desenvolvido pela Sociedade Americana de Psicologia). A opção por tais bases ocorreu em virtude de elas recuperarem a maior parte das publicaçóes realizadas envolvendo estudos com indivíduos diagnosticados com TEA e vinculá-los a competências como a empatia. Durante as buscas, foram empregados os descritores: "Autism", "ASD" (Autism Spectrum Disorder) e "Empathy", em língua inglesa, pois a busca com descritores em português não obteve resultados. Uma das exigências desta revisão era a de que ao menos um dos termos fosse encontrado em algum dos seguintes campos de busca: palavras-chave, título ou resumo. Nesse contexto, os cruzamentos booleanos foram "ASD” OR "Autism" AND "Empathy".

\subsection{Critérios de INCLUSẤo E EXCLUSÃo}

Foram incluídos desta revisão: a) estudos empíricos - correlacionais e de intervenção - que apresentassem pesquisas sobre as relações entre empatia e TEA; b) artigos indexados acerca do tema, publicados em inglês e veiculados entre 2005 e 2020 - optou-se por esse recorte justamente por conta de algumas publicações do período serem citadas de forma recorrente e trazerem contribuiçóes importantes para a discussão que esta revisão de literatura propóe.

Foram excluídos: a) estudos em forma de teses de Doutorado, dissertações de Mestrado e monografias de curso de Graduação; b) artigos cujos participantes não tivessem diagnóstico verificado para o TEA; e c) artigos de revisóes teóricas ou bibliográficas.

\subsection{Procedimentos}

A seleção de artigos ocorreu em dezembro de 2020. Os descritores escolhidos e suas combinações foram utilizados nas bases PubMed, ERIC e PsycINFO apoiados em um acesso anterior à Biblioteca Virtual em Saúde - Psicologia Brasil (BVS-Psi), realizado durante o processo de recuperação do material nas bases de dados. Inicialmente, foram excluídos os artigos repetidos e os que não cumpriram os critérios de inclusão estipulados pela revisão. Em seguida, foi efetivada uma leitura atenta dos resumos dos artigos pré-selecionados para confirmar se eles 
se encaixavam no escopo desta revisão. Após a seleção definitiva, restaram 26 artigos que foram lidos na íntegra e analisados.

\subsection{ANÁlisE DE DADOS}

Os 26 artigos selecionados foram organizados em um arquivo do Excel para análise aprofundada. Os principais elementos analisados foram: o título do estudo, o ano de publicação, os autores, as palavras-chave, o periódico da publicação, o tipo de estudo, a amostra (os participantes), os instrumentos, os objetivos e os seus resultados principais. Além disso, após a análise desses elementos, foram estabelecidas categorias temáticas com a finalidade de discutir as características desses estudos. Assim, os dados obtidos nos trabalhos revisados são discutidos de acordo com as seguintes categorias: a) Empatia afetiva e cognitiva no TEA; b) Diferenças associadas ao gênero na empatia em pessoas com TEA; e c) Diferenças relacionadas à idade e ao QI na empatia em indivíduos com TEA.

Como pode ser observado na Figura 1, a seguir, as investigaçóes iniciais levaram ao seguinte resultado, em termos quantitativos de materiais recuperados nas bases pesquisadas: PubMed $(\mathrm{n}=124)$, ERIC $(\mathrm{n}=31)$ e PsycINFO $(\mathrm{n}=25)$, totalizando 180 registros. Grande parte desses estudos foram excluídos tendo em vista que abordavam questóes que não envolviam a empatia em pessoas com TEA ou eram pesquisas de revisão teórica. Destaca-se que, apesar do elevado número de estudos que foram recuperados nas três bases, a seleção dos últimos 15 anos e os critérios de inclusão desta pesquisa diminuíram consideravelmente o número de publicaçóes elegíveis para esta revisão. 


\section{Figura 1}

Fluxograma do processo de revisão

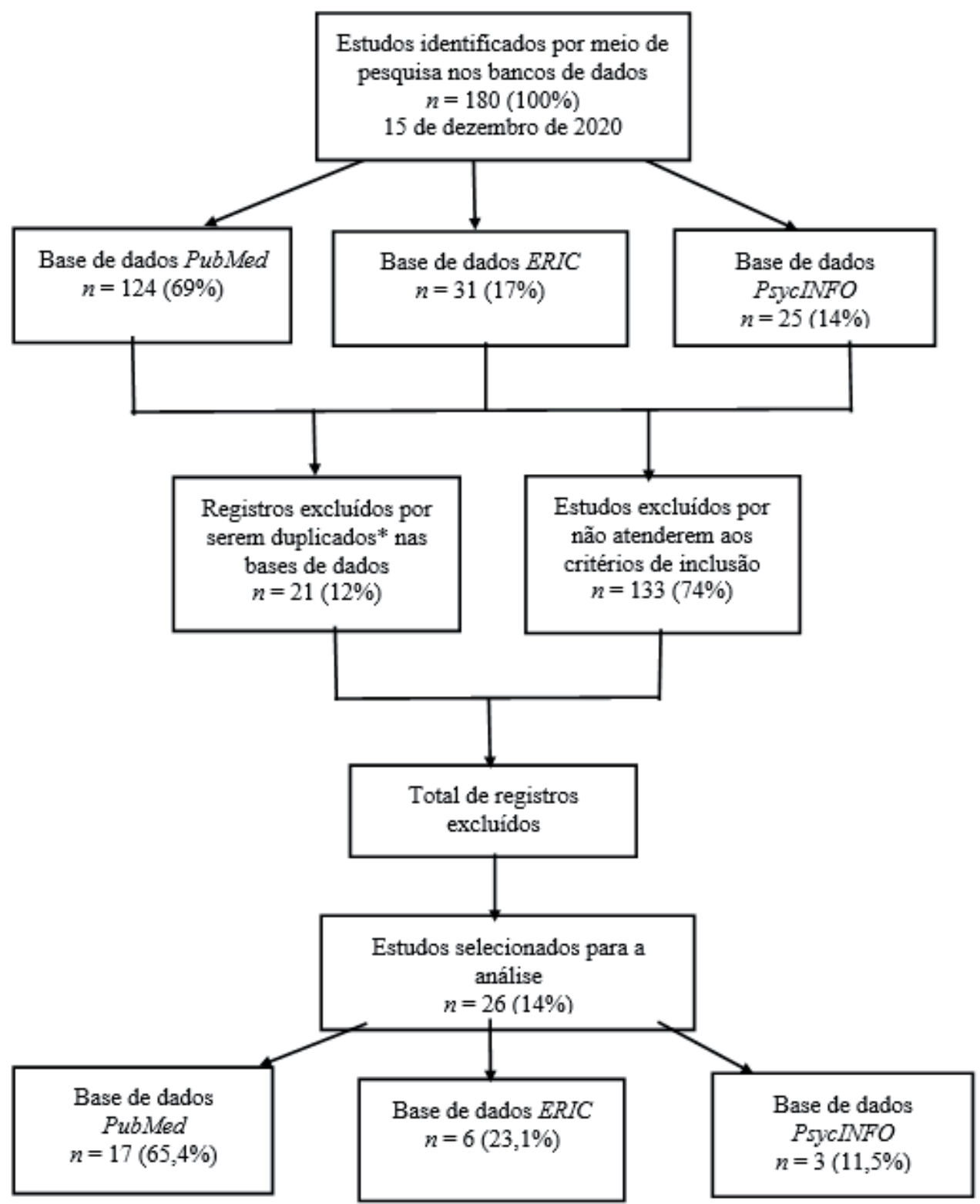

Nota: * 21 estudos duplicados, sendo: 13 registrados no PubMed e no PsycINFO; cinco no ERIC e no PsycINFO e três no PubMed e ERIC. 


\section{Resultados e discussáo}

Após a aplicação dos critérios de inclusão e exclusão elaborados para este estudo e da eliminação das pesquisas repetidas, restaram 26 artigos que se encaixaram nos critérios da revisão, os quais foram lidos na íntegra. Portanto, como anteriormente mencionado, o corpus desta revisão é composto por 26 artigos, o que corresponde a 14\% dos registros de estudos, publicados entre 2005 e 2020, recuperados nas buscas realizadas nas bases eletrônicas. A partir da leitura e da análise inicial dos artigos, foi organizado o Quadro 1 que segue, o qual apresenta os elementos centrais de análise, ou melhor, as principais características dos 26 estudos que compóem esta revisão.

\section{Quadro 1}

Classificação dos artigos selecionados $(n=26)$

\begin{tabular}{|c|c|c|}
\hline $\begin{array}{l}\text { Autores e ano de } \\
\text { publicaçáo }\end{array}$ & Objetivos dos estudos selecionados & $\begin{array}{l}\text { Principais resultados dos estudos } \\
\text { selecionados }\end{array}$ \\
\hline Goldenfeld et al. (2005) & $\begin{array}{l}\text { Verificar diferenças de gênero na empatia em } \\
\text { pessoas com Asperger em comparação com a } \\
\text { população geral. }\end{array}$ & $\begin{array}{l}\text { As mulheres demonstraram maior empatia, } \\
\text { enquanto os homens, maior simpatia. No } \\
\text { entanto, quando os escores foram combinados, } \\
\text { não houve diferença significativa. }\end{array}$ \\
\hline Wheelwright et al. (2006) & $\begin{array}{l}\text { Investigar a empatia em seus componentes } \\
\text { cognitivos e afetivos em estudantes universitá- } \\
\text { rios com TEA. }\end{array}$ & $\begin{array}{l}\text { A posiçáo de um indivíduo no TEA, definido } \\
\text { pelo número de traços autistas, influencia seus } \\
\text { escores de empatia. Quanto maior o grau de } \\
\text { autismo, mais prejudicada é a empatia cognitiva } \\
\text { e afetiva. }\end{array}$ \\
\hline Lombardo et al. (2007) & $\begin{array}{l}\text { Verificar se a empatia e a cogniçấo são prejudi- } \\
\text { cadas em indivíduos com TEA. }\end{array}$ & $\begin{array}{l}\text { Indivíduos com TEA pontuaram mais baixo em } \\
\text { todas as medidas de empatia: cognitiva, afetiva, } \\
\text { tomada de perspectiva e preocupaçáo empática. }\end{array}$ \\
\hline Rogers et al. (2007) & $\begin{array}{l}\text { Examinar a empatia cognitiva e afetiva em } \\
\text { indivíduos com TEA. }\end{array}$ & $\begin{array}{l}\text { Pessoas com TEA pontuaram abaixo da média } \\
\text { em empatia cognitiva, sugerindo dificuldades na } \\
\text { tomada de perspectiva. }\end{array}$ \\
\hline Wakabayashi et al. (2007) & $\begin{array}{l}\text { Testar a teoria da empatia-sistematização das } \\
\text { diferenças de gênero e a teoria do cérebro } \\
\text { masculino extremo do TEA. }\end{array}$ & $\begin{array}{l}\text { As mulheres pontuaram mais alto do que os } \\
\text { homens com TEA em medidas de empatia, } \\
\text { sugerindo perfis diferentes em relação ao gênero } \\
\text { para a distribuiçấo de tipos de cérebro, diferen- } \\
\text { ciando masculino e feminino. }\end{array}$ \\
\hline Clark et al. (2008) & $\begin{array}{l}\text { Verificar como pessoas com TEA analisam } \\
\text { emoçốes a partir de expressóes faciais e se há } \\
\text { alguma perda de compreensão emocional. }\end{array}$ & $\begin{array}{l}\text { Indivíduos com TEA tiveram um desempenho } \\
\text { pior na extraçáo de emoçôes, sem diferenças } \\
\text { para gênero. O déficit no processamento } \\
\text { emocional pode contribuir para as dificuldades } \\
\text { do TEA em mimetismo, empatia e processos } \\
\text { relacionados. }\end{array}$ \\
\hline Dziobek et al. (2008) & $\begin{array}{l}\text { Avaliar a empatia em seus componentes } \\
\text { afetivos e cognitivos em um grupo de adultos } \\
\text { com TEA. }\end{array}$ & $\begin{array}{l}\text { Indivíduos com TEA pontuaram mais baixo do } \\
\text { que o grupo de controle na tarefa de empatia } \\
\text { cognitiva e não pontuaram significativamente } \\
\text { diferente na escala de preocupaçáo empática - } \\
\text { uma medida de empatia emocional. }\end{array}$ \\
\hline
\end{tabular}

Investigar se as diferenças entre os gêneros masculino e feminino encontradas em adultos
com TEA em relaçáo aos componentes da Auyeung et al. (2009) empatia também podem ser observadas em crianças.
Empatia em crianças mostra padróes semelhantes de diferenças de sexo como as observadas em adultos. Crianças com TEA tendem a um perfil "hiper masculinizado", independentemente do sexo. 

com TEA.
Schrandt et al. (2009) Ensinar a resposta empática a quatro crianças

Aumentos na resposta empática ocorreram sistematicamente com a introdução do tratamento em todos os participantes e categorias de resposta.

Investigar os mecanismos neurais de empatia em adolescentes com TEA e explorar as contribuiçóes familiares para correlatos dessa habilidade.

Greimel et al. (2010)

Examinar meninos com TEA em tarefas que

Jones et al. (2010) avaliam aspectos de empatia afetiva e tomada da perspectiva cognitiva dos outros.

Moriwaki et al. (2011)

Comparar as características da empatia como suporte social para a construçấo de amizades em crianças com TEA.

Schulte-Rüther et al. (2011)

Identificar disfunçóes nas redes cerebrais que podem estar subjacentes ao comportamento empático perturbado em pessoas com TEA.
O grupo com TEA mostrou ativação diminuída do giro fusiforme em comparaçâo com os controles. Neuralmente, pais de crianças com TEA também mostraram redução na ativação do giro fusiforme ao inferir as emoçōes dos outros.

Os meninos com TEA tiveram dificuldades com tarefas que requeriam tomada de perspectiva cognitiva, mas relataram experiências emocionais e empatia afetiva com a vítima que estava de acordo com os meninos do grupo controle.

Em testes de simulaçáo afetiva, em 1/3 das crianças com TEA a empatia reativa foi significativamente mais baixa do que o grupo controle, sugerindo dificuldades em construir amizades recíprocas.

Pessoas com TEA tiveram um desempenho igual ao do grupo controle durante a tarefa de avaliar rostos, emocionalmente (outra tarefa), mas mostraram respostas menos congruentes para relatar as emoçóes eliciadas em si mesmas pelas faces emocionais (autotarefa).

Adultos com TEA demonstraram menor reconhecimento de emoçóes, tenderam a julgar os rostos de forma mais negativa, com baixa empatia cognitiva.

Quando as seis emoçốes básicas foram analisadas na comunicação, o grupo com TEA apresentou desempenho prejudicado em cinco das seis expressôes (feliz, triste, raivoso, medo e nojo).

Níveis mais baixos de empatia cognitiva avaliada por pais e professores e níveis semelhantes de empatia afetiva em crianças com TEA.
Avaliar a empatia e o comportamento pró-social em crianças com TEA.
Mudanças diferenciais dependentes da idade foram evidentes no relato de emoçóes no córtex pré-frontal dorsolateral direito, córtex pré-frontal medial direito, córtex parietal inferior direito, ínsula anterior direita e córtex occipital.
Schulte-Rüther et al. (2014)

Comparar as trajetórias de desenvolvimento nos mecanismos neurais subjacentes à empatia em indivíduos com TEA e indivíduos sem TEA.
Adolescentes com TEA apresentaram déficits em todas as medidas de mentalização: eles eram incapazes de interpretar e compreender os estados mentais e emocionais de outras pessoas.

Pessoas com TEA obtiveram pontuação mais baixa em empatia cognitiva, mas tiveram pontuação dentro da faixa média em empatia afetiva.

Adultos com TEA mostraram mais engajamento relacionado à dor empática, juntamente com aumento da atividade neural no córtex insular anterior. 


$\begin{array}{ll}\text { Koehne et al. (2016) } & \begin{array}{l}\text { Investigar o efeito da sincronia interpessoal } \\ \text { unilateral na empatia em duas tarefas simples } \\ \text { de seguir o líder na comunicaçáo virtual em } \\ \text { indivíduos com TEA. }\end{array}\end{array}$

Montgomery et al. (2016)

Examinar, entre os participantes, a capacidade autorrelatada de empatia com os outros e a capacidade de ler estados mentais nos olhos de outras pessoas.

Williams e Cameron
$\begin{array}{ll}\text { (2017) } & \text { Avaliar as propriedades psicométricas da em- } \\ \text { patia em participantes com e sem TEA. }\end{array}$

$\begin{array}{ll}\text { Williams e Cameron } & \text { Avaliar as propriedades psicométricas da } \\ \text { (2017) } & \text { patia em participantes com e sem TEA. }\end{array}$

Mul et al. (2018)

Investigar a intercepção, emoção e empatia, verificando as relações entre esses fatores no TEA.
No grupo com TEA, o grau de sincronia produzida, ou melhor, a capacidade de seguir o líder virtual em uma interação, foi associado a um desempenho de empatia cognitiva superior medido com tarefas de reconhecimento de emoçōes.

Adultos com altos níveis de TEA tiveram um desempenho menor em ler estados mentais. Isso sugere que adultos com TEA podem precisar de mais apoio, particularmente na mentalização e no reconhecimento de emoçóes complexas.

As diferenças individuais nos traços empáticos são explicadas pela variação nos mecanismos que servem à aprendizagem sensório-motora (cognição motora) em relaçáo aos estados emocionais.

Os participantes com TEA mostraram sensibilidade interceptiva (SI) reduzida e consciência interceptiva $(\mathrm{CI})$ reduzida.

Ao comparar os escores de mudança de responsividade empática entre o pré e o pós-teste, o grupo de intervenção teve um desempenho significativamente melhor do que o grupo da lista de espera.

Não houve diferenças sociais cognitivas significativas entre os grupos nas medidas de reconhecimento de emoção e inferência social.
Investigar se o treinamento de Teoria da Mente melhorou a responsividade empática de jovens com TEA quando
observação estruturada.

Holopainen et al. (2018)

a dultos com TEA.

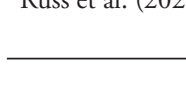

Sumariando os dados apresentados no Quadro 1, verifica-se que o perfil dos participantes selecionados para as amostras dos estudos analisados foi, predominantemente, composto por adultos diagnosticados com TEA $(n=17 ; 65,4 \%)$. Desse grupo, uma parcela considerável dos estudos $(\mathrm{n}=13 ; 76,5 \%)$ focou em ambos os gêneros - masculino e feminino - enquanto uma parte menor $(\mathrm{n}=4 ; 23,5 \%)$ focalizou apenas homens adultos. Ainda em relação à amostra, alguns estudos foram feitos com crianças com TEA ( $n=5 ; 19,2 \%)$ (Auyeung et al., 2009; Deschamps et al., 2014) e outros com adolescentes com TEA ( $\mathrm{n}=4 ; 15,4 \%)$ (Greimel et al., 2010; Mazza et al., 2014). Nessa perspectiva, destaca-se que alguns dos estudos também incluíram os pais como parte da amostra (Auyeung et al., 2009; Deschamps et al., 2014; Greimel et al., 2010; Sucksmith et al., 2013).

Em relação aos objetivos de pesquisa, nota-se um interesse crescente por parte dos pesquisadores em entender como a empatia ocorre em crianças a partir da entrada na vida escolar e o processo de alfabetização, especialmente aos seis anos de idade (Deschamps et al., 2014), em adolescentes, principalmente para averiguar como os componentes cognitivo e afetivo impactam na socializaçáo (Mazza et al., 2014), e em adultos, investigando potenciais diferenças de gênero (Russ et al., 2020). Além disso, alguns estudos focalizaram a busca por diferenças entre homens e mulheres com TEA em relação à empatia (Auyeung et al., 2009; Goldenfeld et al., 2005). Um dos estudos investigou se o treinamento da Teoria da Mente poderia melhorar os níveis de empatia em crianças e adolescentes com TEA (Holopainen et al., 2018), e outra 
pesquisa desenvolveu uma intervenção para ensinar crianças com TEA a demonstrarem habilidades empáticas (Schrandt et al., 2009).

No que tange à mensuração da empatia em indivíduos com TEA, dos 26 estudos elencados, 13 deles - 50\% das pesquisas - utilizaram como principal instrumento o questionário de autoavaliação conhecido como "Quociente de Empatia (QE)" (Baron-Cohen et al., 2004), instrumento desenvolvido para analisar características individuais da empatia (dimensôes cognitiva e afetiva, reconhecimento de emoção e atribuição de intenção). Outro instrumento utilizado em grande parte dos estudos $(n=17,65,4 \%)$ foi o Quociente do Espectro Autista (Baron-Cohen et al., 2001), que mensura não apenas o TEA em altos níveis, mas verifica características dentro de um continuum em níveis mais baixos em toda a população, revelando diferenças individuais dentro do TEA. Além desses instrumentos, outros apontados foram o Índice de Reatividade Interpessoal (Rogers et al., 2007) e o Índice de Empatia de Bryant (Bryant, 1982).

Ademais, como já mencionado, os dados obtidos nos artigos revisados foram analisados a partir de três categorias, que sintetizam os principais achados (resultados) bem como as possibilidades e as limitaçóes dos estudos.

\subsection{EMPATIA AFETIVA E COGNITIVA NO TEA}

A natureza complexa e multidimensional da empatia exige que a pessoa que demonstra empatia compreenda cognitivamente o estado emocional de outra pessoa e que compartilhe a afeição apresentada (Goldenfeld et al., 2005). Apesar de apresentados separadamente, há um consenso entre pesquisadores que, para demonstrar empatia, são necessários os dois componentes, o cognitivo e o afetivo (Wheelwright et al., 2006). Respostas com baixa capacidade empáticas têm sido associadas com o TEA (Lombardo et al., 2007). Isso se deve, principalmente, por prejuízos demonstrados no componente cognitivo da empatia em pessoas diagnosticadas com TEA (Deschamps et al., 2014; Dziobek et al., 2008; Mazza et al., 2014; Moriwaki et al., 2011; Rueda et al., 2015).

Ainda, pesquisas realizadas nas últimas décadas indicam que pessoas com TEA têm dificuldades com o componente cognitivo da empatia (Jones et al., 2010; Wakabayashi et al., 2007). Estudos medindo processos cognitivos e afetivos relacionados à empatia verificaram em adultos com TEA prejuízos na tomada de perspectiva cognitiva dos outros (Dziobek et al., 2008; Mathersul et al., 2013; Sucksmith et al., 2013), bem como redução no componente cognitivo mas não no afetivo - da empatia em crianças com diagnóstico de TEA (Deschamps et al., 2014; Jones et al., 2010). Nessa linha, o estudo de Rueda et al. (2015) demonstrou que o grupo de pessoas com TEA obteve pontuação mais baixa do que os controles em empatia cognitiva, mas apresentou pontuação dentro da faixa média em empatia afetiva. No entanto, o mesmo estudo apontou um déficit no reconhecimento de emoçóes positivas em pessoas com TEA. Esses achados confirmam descobertas anteriores sobre empatia cognitiva e fornecem uma nova visão acerca das habilidades de reconhecimento de emoção nessa população (Rueda et al., 2015).

Já Mazza et al. (2014) descobriram não apenas dificuldades e escore diminuído do componente cognitivo da empatia em adolescentes com TEA, mas também um déficit da empatia afetiva - valência emocional negativa. Dito de outra forma, os adolescentes com TEA 
foram capazes de empatizar com a experiência emocional de outras pessoas quando expressam emoçôes com valência positiva, mas não são capazes de fazê-lo quando a valência emocional é negativa.

O padrão geral de descobertas, a respeito do processamento emocional em TEA, demonstra que indivíduos com autismo tendem a apresentar mais dificuldades em desenvolver o aspecto cognitivo da empatia, mas conseguem manter níveis apropriados de empatia afetiva (Deschamps et al., 2014; Rueda et al., 2015), o que reafirma a hipótese de desequilíbrio dessa habilidade em pessoas com TEA (Smith, 2009). Ainda assim, é importante destacar que a maneira como as emoçóes são processadas no cotidiano está inserida em uma estrutura complexa que inclui fatores sociais, comunicativos e cognitivos dinâmicos. Portanto, quando as emoçóes precisam ser reconhecidas e expressas em interaçóes sociais, os comportamentos emocionais de indivíduos com TEA demonstram vulnerabilidade em relação ao componente cognitivo da empatia (Russ et al., 2020).

\subsection{DIFERENÇAS ASSOCIADAS AO GÊNERO NA EMPATIA EM PESSOAS COM TEA}

Um dos fatores de risco para um diagnóstico de TEA é o gênero, pois os homens são quatro vezes mais suscetíveis a serem diagnosticados com alguma forma de autismo do que as mulheres (Fombone, 2009). Estudos que analisaram perfis de sistematização da empatia em crianças, adolescentes e adultos com TEA apontaram que esses indivíduos tendem a ter um perfil predominantemente masculinizado, e que os homens demonstram déficits em empatia de forma geral e, no componente cognitivo, de maneira específica (Auyeung et al., 2009; Wakabayashi et al., 2007).

Wakabayashi et al. (2007), ao pesquisarem uma amostra de universitários, descobriram que mulheres com TEA pontuaram em um nível muito mais alto do que os homens com TEA na empatia afetiva e cognitiva. Jones et al. (2010) apontaram que meninos com TEA têm grandes dificuldades na tomada de perspectiva cognitiva dos outros, demonstrando déficits para o gênero masculino nesse componente da empatia. Já Auyeung et al. (2009) descobriram padrôes semelhantes de diferenças de gênero em crianças, como já observado em adultos, demonstrando uma prevalência de menos empatia no gênero masculino e um perfil hiper masculinizado em crianças com TEA.

Greimel et al. (2010) verificaram, em estudo feito apenas com meninos e seus pais, que os pais de meninos com TEA também demonstraram déficits na inferência de estados emocionais, assim como os seus filhos, quando em comparação com o grupo controle. Os resultados do estudo trazem implicações para o Fenótipo Ampliado do Autismo, pois os pais de crianças com TEA também mostraram ativação reduzida do giro fusiforme ao inferir as emoçóes dos outros. Esses achados na ativação do giro fusiforme em adolescentes e parentes de primeiro grau sugerem que a disfunção constitui um desvio no TEA. Ademais, os resultados fornecem evidências de que a face neural e os mecanismos de espelhamento estão implicados em déficits de empatia no TEA (Greimel et al., 2010).

Apesar de grande parte das pesquisas indicar um déficit masculino na empatia, especialmente no componente cognitivo, alguns estudos mostram resultados diferentes (Clark et al., 2008; Goldenfeld et al., 2005). Goldenfeld et al. (2005) revelaram que, apesar das mu- 
lheres demonstrarem mais empatia cognitiva e afetiva, nos valores gerais dos testes aplicados, não houve diferença significativa do ponto de vista estatístico para a comparação entre gênero. Seguindo essa linha, Clark et al. (2008) também não encontraram diferenças significativas para medidas de empatia em comparaçóes de gênero em indivíduos com TEA.

Assim, diante dos resultados apontados, identifica-se uma predominância masculina no déficit de empatia, especialmente no componente cognitivo. Entretanto, alguns estudos não apontam diferenças significativas entre gêneros (Clark et al., 2008; Goldenfeld et al., 2005). Uma das explicaçóes para essa disparidade é a de que as diferenças de gênero associadas ao comportamento social, como é o caso da empatia, podem ser atribuídas aos papéis de gênero tradicionais, pois espera-se que as mulheres, em geral, mostrem habilidades de cuidado social mais fortes do que os homens; somando-se a isso, deve-se relembrar que a prevalência de diagnósticos de TEA é muito mais elevada na população masculina (Fombone, 2009).

\subsection{DIFERENÇAS RELACIONADAS À IDADE E AO QI NA EMPATIA EM INDIVÍDUOS COM TEA}

O desenvolvimento da empatia é um processo gradativo que tem o seu início na infância, avançando cognitivamente ao longo da adolescência e vida adulta (Williams \& Cameron, 2017). Em relação ao QI, as pesquisas sobre TEA e empatia apontaram a importância de diferenciar os quadros de diagnóstico para execução do estudo (Mul et al., 2018; Rueda et al., 2015). Por exemplo, os estudos feitos com indivíduos com Asperger exigiam um nível intelectual semelhante entre as amostras (Goldenfeld et al., 2005; Rueda et al., 2015). Nesse sentido, outros estudos parearam um grupo de adultos com TEA de alto funcionamento com um grupo de adultos com síndrome de Asperger (Montgomery et al., 2016) ou um grupo de adultos com TEA com um grupo de adultos com desenvolvimento típico (Mul et al., 2018), para que os escores de empatia pudessem ser analisados de forma mais aproximada, pois a empatia é uma competência aprendida socialmente e que tem relação com habilidades cognitivas.

Alguns estudos investigaram o ensino da empatia para crianças em idade escolar (Holopainen et al., 2018; Schrandt et al., 2009). Schrandt et al. (2009), por exemplo, enfocou seu estudo no ensino de respostas empáticas a crianças por meio de vinhetas com bonecos que demonstravam vários tipos de emoçóes. Os resultados apontaram que aumentos na resposta empática ocorreram sistematicamente com a introdução do tratamento em todos os participantes e em todas as categorias de resposta. Holopainen et al. (2018) desenvolveram uma intervenção para crianças com TEA visando o treinamento da Teoria da Mente como uma forma de ensinar responsividade empática. As sessóes sempre tiveram a mesma estrutura: discussão, exercícios, resumo da reunião para os pais e apresentação de dever de casa. Além disso, focaram tópicos como reconhecimento de emoção, fingimento, crença falsa e humor. $\mathrm{O}$ treinamento foi ministrado em um centro psiquiátrico, para cinco ou seis crianças simultaneamente. Nos grupos, a diferença mútua de idade não ultrapassou três anos. As sessóes foram supervisionadas por um clínico certificado que recebeu treinamento para a intervenção. $O$ grupo que passou pela intervenção de Teoria da Mente demonstrou melhoras na compreensão empática ao final do processo.

Moriwaki et al. (2011) verificaram em sua investigação que os escores de Empatia Reativa de mais de um terço das crianças com autismo foram visivelmente mais baixos do que 
os das crianças com desenvolvimento típico. Isso sugere que uma fraqueza na Empatia Reativa em crianças com TEA pode ser um fator importante na dificuldade que elas têm em construir relacionamentos recíprocos com amigos. Deschamps et al. (2014) também descobriram prejuízos na empatia cognitiva em crianças com TEA, e que esses prejuízos impactaram suas relaçóes familiares e escolares.

Sobre o desenvolvimento da adolescência até a vida adulta, Schulte-Rüther et al. (2014) investigaram as trajetórias neurais da empatia em indivíduos com TEA com idades entre 12 e 31 anos de idade. De acordo com os autores, as modulaçóes das áreas cerebrais dependentes da idade podem refletir o ajuste fino das redes corticais pela redução da atividade cerebral inespecífica para a tarefa. Assim, conforme a idade avança, a empatia vai se tornando melhor, mesmo que menor em comparação com a população sem TEA. Mathersul et al. (2013) verificaram que a baixa empatia cognitiva era uma característica demonstrada por adultos com TEA. No estudo conduzido por Sucksmith et al. (2013), homens e mulheres com TEA obtiveram pontuaçóes significativamente mais baixas nas medidas de empatia do que os de controles. Quando as seis emoções básicas distintas foram analisadas separadamente, o grupo com TEA apresentou desempenho prejudicado em cinco das seis expressóes (felicidade, tristeza, zangado, medo e nojo).

\section{Conclusóes}

Em face do resgate empreendido por esta revisão de estudos desenvolvidos entre 2005 e 2020, destaca-se que a pesquisa atual sobre empatia em indivíduos com TEA é um campo em construção e possui estudos que caracterizam os efeitos de gênero, idade, QI e características do ambiente social. Nesse panorama, é imprescindível uma abordagem multidimensional, que possa explicar e diferenciar os componentes cognitivo e afetivo da empatia em pessoas com TEA, em busca de intervir e desenvolver as habilidades que aparecem como mais vulneráveis nessa população. Vários estudos mostraram que a empatia cognitiva, que envolve inferir a emoção dos outros, pode ser reduzida no TEA, enquanto a empatia afetiva (a capacidade de compartilhar a emoção dos outros) pode não ser tão adversamente afetada.

Contudo, é importante salientar que esta revisão apresenta uma limitação no que se refere aos descritores utilizados para a busca de artigos, pois, entre os anos 2005 e 2013, o autismo foi descrito mais como "Autistic Disorder" e "Pervasive Developmental Disorder" em detrimento de "ASD", que passou a ser mais corrente na literatura após o ano de 2013, com a publicação do DSM-5 (APA, 2014). Nesse sentido, sugere-se que revisóes futuras abarquem tais descritores como parte do processo de busca por artigos, caso utilizem o mesmo recorte temporal. Além disso, esta revisão focalizou seus esforços em pesquisar a empatia, mas outros pesquisadores podem, também, incluir a Teoria da Mente como parte dos descritores caso queiram explorar para além do conceito da empatia.

De outra parte, as limitaçóes dos estudos revisados envolvem a complexidade das demandas das tarefas e dos testes utilizados e a natureza do contexto social dos indivíduos com TEA. De qualquer forma, o conhecimento que emerge dos resultados das pesquisas sublinha um déficit no componente cognitivo da empatia, que não é demonstrado pelo aspecto afetivo dessa mesma habilidade em pessoas com TEA. Essa informação pode ajudar a desenvolver 
estratégias e intervençóes para (re)direcionar as dificuldades relacionadas às emoçóes, com a finalidade de melhorar a vida emocional de indivíduos com TEA.

Além disso, identificou-se uma expressiva lacuna nos estudos envolvendo TEA e empatia em contextos (países) que não o norte-americano. Nesse viés, sugere-se que, no Brasil, sejam empreendidos esforços por parte dos pesquisadores para alavancar as investigaçóes na área da empatia, notadamente em pessoas com TEA, dada sua importância na integração (adaptação) e na comunicação social. Em outras palavras, trabalhar com essa temática mostra-se uma agenda de pesquisa tanto promissora quanto necessária na sociedade atual - em que as habilidades socioemocionais têm sido cada vez mais exigidas.

Considera-se, ainda, que, entender melhor as relaçóes entre empatia afetiva e cognitiva em pessoas com TEA pode lançar luz sobre práticas clínicas, educativas e de pesquisa no sentido de contribuir com a melhoria de diagnósticos, atendimentos e terapias. Assim, uma das formas de contribuir com a Educação Especial brasileira é trazer novas perspectivas e realizar um exame crítico da empatia em pessoas com diagnóstico de TEA, no intuito de desvelar os mecanismos responsáveis pelos déficits empáticos.

\section{REFERÊNCIAS}

Associação Americana de Psiquiatria. (2014). DSM-V-TR: Manual diagnóstico e estatístico de transtornos mentais. Artmed.

Auyeung, B., Wheelwright, S., Allison, C., Atkinson, M., Samarawickrema, N., \& Baron-Cohen, S. (2009). The children's empathy quotient and systemizing quotient: Sex differences in typical development and in autism spectrum conditions. Journal of Autism and Developmental Disorders, 39(11), 1509-1521. https://doi.org/10.1007/s10803-009-0772-x

Baron-Cohen, S., \& Wheelwright, S. (2004). The empathy quotient: an investigation of adults with Asperger Syndrome or high functioning autism, and normal sex differences. Journal of Autism and Developmental Disorders, 34, 163-175. https://doi.org/10.1023/B:JADD.0000022607.19833.00.

Baron-Cohen, S., Wheelwright, S., Skinner, R., Martin, J., \& Clubley, E. (2001). The autism spectrum quotient (AQ): evidence from Asperger syndrome/high functioning autism, males and females, scientists and mathematicians. Journal of Autism and Developmental Disorders, 31, 5-17. https:// doi.org/10.1023/A:1005653411471

Blair, R. J. R. (2005). Responding to the emotions of others: Dissociating forms of empathy through the study of typical and psychiatric populations. Consciousness \& Cognition, 14, 698-718. https://doi. org/10.1016/j.concog.2005.06.004

Bryant, B. K. (1982). An index of empathy for children and adolescents. Child Development, 53(2), 413425. https://doi.org/10.2307/1128984

Clark, T. F., Winkielman, P., \& McIntosh, D. N. (2008). Autism and the extraction of emotion from briefly presented facial expressions: Stumbling at the first step of empathy. Emotion, 8(6), 803-809. https://doi.org/10.1037/a0014124

Davis, M. H. (1980). A multidimensional approach to individual differences in empathy. JSAS Catalog of Selected Documents in Psychology, 10, 85-103. 
Decety, J., \& Jackson, P. L. (2004). The functional architecture of human empathy. Behavioral and Cognitive Neuroscience Reviews, 3, 71-100. https://doi.org/10.1177/1534582304267187

Deschamps, P. K. H., Been, M., \& Matthys, W. (2014). Empathy and empathy induced prosocial behavior in 6- and 7-year-olds with autism spectrum disorder. Journal of Autism and Developmental Disorders, 44(7), 1749-1758. https://doi.org/10.1007/s10803-014-2048-3

Domingues, S. F. S., \& Maluf, M. R. (2008). Compreendendo estados mentais: procedimentos de pesquisa a partir da tarefa original de crença falsa. In T. M. Sperb, \& M. R. Maluf (Eds.), Desenvolvimento sociocognitivo: estudos brasileiros sobre Teoria da Mente (1a ed., pp. 11-31). Vetor.

Dziobek, I., Rogers, K., Fleck, S., Bahnemann, M., Heekeren, H. R., Wolf, O. T., \& Convit, A. (2008). Dissociation of cognitive and emotional empathy in adults with Asperger syndrome using the Multifaceted Empathy Test (MET). Journal of Autism and Developmental Disorders, 38, 464-473. https://doi.org/10.1007/s10803-007-0486-x

Fletcher-Watson, S., McConnell, F., Manola, E., \& McConachie, H. (2014). Interventions based on the Theory of Mind cognitive model for autism spectrum disorder (ASD). Cochrane Database Systematic Reviews, 3:CD008785, 1-35. https://doi.org/10.1002/14651858.cd008785.pub2

Fombonne, E. (2009). Epidemiology of pervasive developmental disorders. Pediatric Research, 65(6), 591-598. https://doi.org/10.1203/pdr.0b013e31819e7203

Gaigg, S. B. (2012). The interplay between emotion and cognition in autism spectrum disorder: implications for developmental theory. Frontiers in Integrative Neuroscience, 6(113), 1-35. https:// dx.doi.org/10.3389\%2Ffnint.2012.00113

Goldenfeld, N., Baron-Cohen, S., \& Wheelwright, S. (2005). Empathizing and systemizing in males, females and autism. Clinical Neuropsychiatry, 2(6), 338-345.

Greimel, E., Schulte-Rüther, M., Kircher, T., Kamp-Becker, I., Remschmidt, H., Fink, G. R., HerpertzDahlmann, B., \& Konrad, K. (2010). Neural mechanisms of empathy in adolescents with autism spectrum disorder and their fathers. NeuroImage, 49(1), 1055-1065. https://doi.org/10.1016/j. neuroimage.2009.07.057

Gu, X., Eilam-Stock, T., Zhou, T., Anagnostou, E., Kolevzon, A., Soorya, L., Hof, P. R., Friston, K. J., \& Fan, J. (2015). Autonomic and brain responses associated with empathy deficits in autism spectrum disorder. Human Brain Mapping, 36, 3323-3338. https://doi.org/10.1002/hbm.22840

Hoffman, M. L. (2000). Empathy and moral development: Implications for caring and justice. Cambridge University Press. https://doi.org/10.1017/CBO9780511805851

Holopainen, A., de Veld, D. M. J., Hoddenbach, E., \& Begeer, S. (2018). Does theory of mind training enhance empathy in autism? Journal of Autism and Developmental Disorders, 49, 3965-3972. https://doi.org/10.1007/s10803-018-3671-1

Jones, A. P., Happé, F. G. E., Gilbert, F., Burnett, S., \& Viding, E. (2010). Feeling, caring, knowing: Different types of empathy deficit in boys with psychopathic tendencies and autism spectrum disorder. The Journal of Child Psychology and Psychiatry, 51(11), 1188-1197. https://doi. org/10.1111/j.1469-7610.2010.02280.x

Koehne, S., Hatri, A., Cacioppo, J. T., \& Dziobek, I. (2016). Perceived interpersonal synchrony increases empathy: Insights from autism spectrum disorder. Cognition, 146, 8-15. https://doi.org/10.1016/j. cognition.2015.09.007 
Lombardo, M. V., Barnes, J. L., Wheelwright, S. J., \& Baron-Cohen, S. (2007). Self-referential cognition and empathy in Autism. Plos One, 2(9), 1-11. https://doi.org/10.1371/journal.pone.0000883

Mathersul, D., McDonald, S., \& Rushby, J. A. (2013). Autonomic arousal explains social cognitive abilities in high-functioning adults with autism spectrum disorder. International Journal of Psychophysiology, 89, 475-482. https://doi.org/10.1016/j.ijpsycho.2013.04.014

Mazza, M., Pino, M. C., Mariano, M., Tempesta, D., Ferrara, M., De Berardis, D., Masedu, F., \& Valenti, M. (2014). Affective and cognitive empathy in adolescents with autism spectrum disorder. Frontiers in Human Neuroscience, 8(791), 1-6. https://doi.org/10.3389/fnhum.2014.00791

Mendes, K. S., Silveira, R. C. C. P., \& Galvão, C. M. (2008). Revisão integrativa: método de pesquisa para a incorporação de evidências na saúde e na enfermagem. Texto-Contexto Enfermagem, 17(4), 758-764. https://doi.org/10.1590/S0104-07072008000400018

Montgomery, C. B., Allison, C., Lai, M.-C., Cassidy, S., Langdon, P. E., \& Baron-Cohen, S. (2016). Do adults with high functioning autism or Asperger syndrome differ in empathy and emotion recognition? Journal of Autism and Developmental Disorders, 46, 1931-1940. https://doi. org/10.1007/s10803-016-2698-4

Moriwaki, A., Ryoko, I., \& Hiroshi, F. (2011). Characteristics of empathy for friendship in children with high-functioning autism spectrum disorders. Japanese Journal of Special Education, 48(6), 593604. https://doi.org/10.6033/tokkyou.48.593

Mul, C. L., Stagg, S. D., Herbelin, B., \& Aspell, J. E. (2018). The feeling of me feeling for you: interoception, alexithymia and empathy in autism. Journal of Autism and Developmental Disorders, 48, 2953-2967. https://doi.org/10.1007/s10803-018-3564-3

Pavarini, G., \& Souza, D. H. (2010). Teoria da mente, empatia e motivação pró-social em crianças pré-escolares. Psicologia em Estudo, 15(3), 613-622. https://doi.org/10.1590/S141373722010000300019

Powell, P. A., \& Roberts, J. (2017). Situational determinants of cognitive, affective, and compassionate empathy in naturalistic digital interactions. Computers in Human Behavior, 68, 137-148. https:// doi.org/10.1016/j.chb.2016.11.024

Rogers, K., Dziobek, I., Hassenstab, J., Wolf, O. T., \& Convit, A. (2007). Who cares? Revisiting empathy in Asperger syndrome. Journal of Autism and Developmental Disorders, 37, 709-715. https://doi. org/10.1007/s10803-006-0197-8

Rueda, P., Fernández-Berrocal, P., \& Baron-Cohen, S. (2015). Dissociation between cognitive and affective empathy in youth with Asperger Syndrome. European Journal of Developmental Psychology, 12(1), 85-98. https://doi.org/10.1080/17405629.2014.950221

Russ, V., Kovshoff, H., Brown, T., Abbott, P., \& Hadwin, J. A. (2020). Exploring the role of empathy in understanding the social-cognitive profile for individuals referred for Autism Spectrum Disorders assessment in adulthood. Journal of Autism and Developmental Disorders, 50, 1470-1478. https:// doi.org/10.1007/s10803-018-3693-8

Schrandt, J. A., Townsend, D. B., Poulson, C. L., \& Carr, J. (2009). Teaching empathy skills to children with autism. Journal of Applied Behavior Analysis, 42(1), 17-32. https://doi.org/10.1901/ jaba.2009.42-17

Schulte-Rüther, M., Greimel, E., Markowitsch, H. J., Kamp-Becker, I., Remschmidt, H., Fink, G. R., \& Piefke, M. (2011). Dysfunctions in brain networks supporting empathy: An fMRI 
study in adults with autism spectrum disorders. Social Neuroscience, 6(1), 1-21. https://doi. org/10.1080/17470911003708032

Schulte-Rüther, M., Greimel, E., Piefke, M., Kamp-Becker, I., Remschmidt, H., Fink, G. R., HerpertzDahlmann, B., \& Konrad, K. (2014). Age-dependent changes in the neural substrates of empathy in autism spectrum disorder. Social Cognitive and Affective Neuroscience, 9(8), 1118-1126. https:// dx.doi.org/10.1093\%2Fscan\%2Fnst088

Shamay-Tsoory, S. G. (2011). The neural bases for empathy. Neuroscientist, 17, 18-24. https://doi. org/10.1177/1073858410379268

Smith, A. (2009). The empathy imbalance hypothesis of autism: a theoretical approach to cognitive and emotional empathy in autistic development. The Psychological Record, 59, 273-294. https://doi. org/10.1007/BF03395663

Sucksmith, E., Allison, C., Baron-Cohen, S., Chakrabarti, B., \& Hoekstra, R. A. (2013). Empathy and emotion recognition in people with autism, first-degree relatives, and controls. Neuropsychologia, 51, 98-105. https://doi.org/10.1016/j.neuropsychologia.2012.11.013

Vignemont, F., \& Singer, T. (2006). The empathic brain: how, when and why? Trends in Cognitive Science, 10(10), 435-441. https://doi.org/10.1016/j.tics.2006.08.008

Wakabayashi, A., Baron-Cohen, S., Uchiyama, T., Yoshida, Y., Kuroda, M., \& Wheelwright, S. (2007). Empathizing and systemizing in adults with and without autism spectrum conditions: Crosscultural stability. Journal of Autism and Developmental Disorders, 37(10), 1823-1832. https://doi. org/10.1007/s10803-006-0316-6

Wheelwright, S., Baron-Cohen, S., Goldenfeld, N., Delaney, J., Fine, D., Smith, R., Weila, L., \& Wakabayashic, A. (2006). Predicting autism spectrum quotient (AQ) from the systemizing quotient-revised (SQ-R) and empathy quotient (EQ). Brain Research, 1079(1), 47-56. https://doi. org/10.1016/j.brainres.2006.01.012

Williams, J. H. G., \& Cameron, I. M. (2017). The actions and feelings questionnaire in autism and typically developed adults. Journal of Autism and Developmental Disorders, 47, 3418-3430. https:// doi.org/10.1007/s10803-017-3244-8

Zaki, J., \& Ochsner, K. N. (2012). The neuroscience of empathy: progress, pitfalls and promise. Nature Neuroscience, 15, 675-680. https://doi.org/10.1038/nn.3085-

Recebido em: 14/01/2021

Reformulado em: 25/05/2021

Aprovado em: 20/07/2021 
ROZA, S.A. \& GUIMARÃES, S.R.K. 